\title{
Vasopressin $V_{2}$ Receptor Antagonist Tolvaptan Is Effective in Heart Failure Patients With Reduced Left Ventricular Systolic Function and Low Blood Pressure
}

\author{
Satoshi Suzuki, ${ }^{1}$ MD, Akiomi Yoshinisa, ${ }^{1}$ MD, Takayoshi Yamaki, ${ }^{1}$ MD, Koichi Sugimoto,,${ }^{1}$ MD, \\ Hiroyuki KuniI, ${ }^{1}$ MD, Kazuhiko NaKazAto, ${ }^{1}$ MD, Yukihiko Abe, ${ }^{2}$ MD, Tomiyoshi Saito, ${ }^{3}$ MD, \\ Takayuki Ohwada, ${ }^{4}$ MD, Hitoshi Suzuki, ${ }^{1}$ MD, Shu-ichi Saitoh, ${ }^{1}$ MD, Isao Kubota,,${ }^{5}$ MD, \\ and Yasuchika TAKEISHI, ${ }^{1} \mathrm{MD}$, on behalf of the AVCMA investigators
}

\begin{abstract}
SUMMARY
Diuresis is a major therapy for the reduction of congestive symptoms in acute decompensated heart failure (ADHF) patients. Carperitide has natriuretic and vasodilatory effects, and tolvaptan produces water excretion without electrolyte excretion. We previously reported the usefulness of tolvaptan compared to carperitide in ADHF patients with fluid volume retention. The purpose of this study was to examine whether the efficacy of tolvaptan was altered in ADHF patients with reduced left ventricular systolic function and in those with hypotension. A total of 109 hospitalized ADHF patients were randomly assigned to either a tolvaptan or a carperitide treatment group. Baseline clinical characteristics were not different between the two groups. We divided these patients based on the left ventricular ejection fraction (EF) by echocardiography, and blood pressure (BP) at the time of admission. Daily urine volume between the tolvaptan and carperitide groups in patients with preserved $\mathrm{EF}(\geq 50 \%)$ was not different, however, in those with reduced $\mathrm{EF}(<50 \%)$, the urine volume was significantly higher in the tolvaptan group than in the carperitide group (day 2, 3, 4, $P<0.05$ for all). Daily urine volume did not differ between these two groups in the high blood pressure group (BP $\geq 140 \mathrm{mmHg}$ ), but was significantly higher in the tolvaptan group than in the carperitide group (day $1, P=0.021$; day $3, P=0.017$ ) in the low blood pressure group $(\mathrm{BP}<140 \mathrm{mmHg}$ ). The present study reveals that tolvaptan is more effective than carperitide, especially in ADHF patients with reduced left ventricular systolic function and without hypertension. (Int Heart J 2015; 56: 213-218)
\end{abstract}

Key words: Diuretics, Carperitide, Heart failure with reduced ejection fraction, Urine volume

$\mathrm{I}^{\mathrm{s}}$ acute decompensated heart failure (ADHF), reduction of excessive fluid volume leads to marked improvement in the signs and symptoms of congestion. ${ }^{1)}$ Diuretics are usually the first-line therapy and are most frequently used for $\mathrm{ADHF}$ treatment, but have a possibility of worsening the renal function and prognosis of heart failure patients. ${ }^{2-5)}$ Carperitide, which is a recombinant of human atrial natriuretic peptide and produces vasodilation and natriuretic effects, is used frequently $(58.2 \%)$ in Japan according to a large-scale multicenter cohort study; ATTEND registry. ${ }^{6}$ Several reports revealed the efficacy and safety of carperitide in acute phase treatment and improvement of long-term prognosis after administration of carperitide in patients with heart failure. ${ }^{7,8)}$ Tolvaptan, which is an orallyadministered selective vasopressin $\mathrm{V}_{2}$ receptor antagonist that promotes aquaresis, ${ }^{9)}$ improves signs and symptoms in ADHF patients without serious adverse events. ${ }^{10)}$ We previously dem- onstrated that tolvaptan showed less blood pressure reduction and higher daily urine volume than carperitide in ADHF patients in an acute heart failure volume control multicenter randomized (AVCMA) trial. ${ }^{11)}$

ADHF patients are typically divided into two groups based on the left ventricular systolic function; heart failure with preserved ejection fraction $(\mathrm{HFpEF})$ and heart failure with reduced ejection fraction (HFrEF). Recent reports revealed a high prevalence of $\mathrm{HFpEF}$, and these patients were characterized as more likely to be older, female, and hypertensive, and less likely to have prior myocardial infarction or history of heart failure. ${ }^{12,13)}$ Although in-hospital mortality is less in $\mathrm{HFpEF}$ patients than in HFrEF patients, HFpEF patients have a similar post-discharge mortality risk and a high rate of rehospitalization equal to that of HFrEF patients. ${ }^{12,13)}$ Interestingly, non-intravenous diuretics were more frequently admin-

From the ${ }^{1}$ Department of Cardiology and Hematology, Fukushima Medical University, ${ }^{2}$ Department of Cardiology, Ohara Medical Center, ${ }^{3}$ Second Department of Medicine, Shirakawa Kosei General Hospital, ${ }^{4}$ Department of Cardiology, Fukushima Red Cross Hospital, Fukushima, and ${ }^{5}$ First Department of Internal Medicine, Yamagata University School of Medicine, Yamagata, Japan.

This study was supported in part by a grant from the Japanese Heart Foundation (No. 12100026).

Address for correspondence: Satoshi Suzuki, MD, Department of Cardiology and Hematology, Fukushima Medical University, 1 Hikarigaoka, Fukushima 960-1295, Japan. E-mail: ssatoshi@fmu.ac.jp

Received for publication July 30, 2014. Revised and accepted September 16, 2014

Released in advance online on J-STAGE February 23, 2015.

All rights reserved by the International Heart Journal Association. 
istered in HFrEF patients than in HFpEF patients, however, the opposite was true with intravenous diuretics. ${ }^{13)}$

Several reports have revealed that clinical profiles and outcomes of ADHF patients were dependent upon systolic blood pressure on admission, which was recently seen as the most important predictor of morbidity and mortality in ADHF patients. ${ }^{14,15)}$ Based on these reports, Mebazaa, et al suggested separating ADHF patients into 5 groups according to clinical scenarios 1 to 5, as these criteria were important for characterizing and treating ADHF patients. ${ }^{16)}$

However, the difference in diuretic effects between tolvaptan and carperitide that depends on either left ventricular systolic function or blood pressure has yet to be elucidated. The aim of this study was to identify the characteristics of patients who gained more benefits from tolvaptan based on the results of the AVCMA trial.

\section{Methods}

Study design: The AVCMA trial was a multicenter, randomized study to determine the efficacy and safety of tolvaptan compared with carperitide in ADHF patients. ${ }^{11)}$ This study was registered in the University Hospital Medical Information Network (ID, 000006258). The first patient was enrolled in January 2011, and a total of 109 patients were randomized by May 2012. Written informed consent was obtained from all study subjects. The study protocol was approved by the ethical committee of Fukushima Medical University and each participating institution in compliance with the Declaration of Helsinki. Sample size estimation was described in a previous report. ${ }^{11)}$

Study participants: Patients were eligible for enrollment if they had presented with fluid volume retention with ADHF or acute exacerbation of chronic heart failure (CHF), and had been diagnosed on the basis of the presence of at least one of the following subjective symptoms; dyspnea, orthopnea, or leg edema, and one of the following signs; rales, peripheral edema, ascites, or pulmonary vascular congestion on chest X-ray. Patients with acute myocardial infarction, severe hypotension (cardiogenic shock), anuria, hypernatremia ( $\mathrm{Na}>147 \mathrm{mEq} / \mathrm{L}$ ), or those who did not feel thirsty or had difficulties with water intake, were excluded.

Our study protocol was reported previously. ${ }^{11)}$ The eligible patients underwent the standard initial treatment, and were randomly assigned into one of two groups consisting of oral administration of tolvaptan or continuous intravenous infusion of carperitide. Echocardiography and blood sample examinations were performed before administration of tolvaptan or carperitide. Left ventricular ejection fraction (EF) was calculated by a modified Simpson method. Heart rate, blood pressure, daily urine volume, and daily volumes of water intake and infusion solution were measured before and after administration of tolvaptan or carperitide. The primary endpoint was the increase in urine volume.

We divided all study subjects into two groups based on the left ventricular EF; patients with left ventricular EF of 50\% or more were assigned to the HFpEF group and those with left ventricular EF of less than $50 \%$ to the HFrEF group. Moreover, we divided all study subjects into two groups based on blood pressure on admission and whether intravenous vasodilators were used. The borderline between clinical scenarios 1 and 2 is $140 \mathrm{mmHg}$ of systolic blood pressure, therefore, we used this criteria and divided the subjects into the high blood pressure group (systolic blood pressure $\geq 140 \mathrm{mmHg}$ ), and the low blood pressure group (systolic blood pressure $<140$ $\mathrm{mmHg}$ ). The intravenous vasodilators chosen were nitrate and Ca-blocker.

Definition of adverse events: In the period of hospitalization, we checked all adverse events that required the change or discontinuation of tolvaptan or carperitide. Evaluation criteria of adverse events were defined in our previous report. ${ }^{11}$

Statistical analysis: Results are expressed as the mean \pm standard deviation (SD), and skewed variables are presented as the median and interquartile range. A $P$ value of 0.05 was considered statistically significant, but no adjustment was made for multiplicity. Significance between the two groups was determined by the unpaired Student's $t$-test for continuous variables and by the chi-square test for discrete variables. The changes in blood pressure, heart rate, daily urine volume, daily volumes of water intake and infusion solution, blood sample data, and echocardiographic data from baseline within the same group were determined by the paired $t$-test. If data were not distributed normally, the Mann-Whitney $U$ test was used. If any data were missing for a particular patient, the entire dataset for that patient was excluded. We used Fisher's exact probability test for the evaluation of adverse events. Statistical analysis was performed with a standard statistical program package (SPSS ver. 21.0, IBM, Armonk, NY, USA).

\section{Results}

Comparisons of daily urine volume based on left ventricular systolic function: The tolvaptan group was comprised of 25 HFpEF patients and $29 \mathrm{HFrEF}$ patients, while the carperitide group consisted of $17 \mathrm{HFpEF}$ patients and $37 \mathrm{HFrEF}$ patients (one patient could not undergo cardiac echocardiography before treatment). As shown in Table I, heart rate, plasma B-type natriuretic peptide level, and left ventricular end-diastolic diameter were significantly lower in HFpEF patients than in HFrEF patients in both the tolvaptan and carperitide groups.

The proportion of ischemic heart disease was higher in HFrEF patients $(20,30 \%)$ than in HFpEF patients $(6,14 \%)$, but there was no significant difference between the tolvaptan $(9,31 \%)$ and carperitide groups $(11,30 \%)$ in $\mathrm{HFrEF}$ patients (Table I). As shown in Figure 1A, the daily urine volume was not significantly different between the tolvaptan and carperitide groups in HFpEF patients. However, the daily urine volume on days 2,3 , and 4 was significantly higher in the tolvaptan group than in the carperitide group in HFrEF patients $(2284 \pm 1034 \mathrm{~mL}$ versus $1763 \pm 651 \mathrm{~mL}$ on day $2 ; 2090 \pm 813$ $\mathrm{mL}$ versus $1675 \pm 660 \mathrm{ml}$ on day 3; $2065 \pm 902 \mathrm{~mL}$ versus $1616 \pm 763 \mathrm{~mL}$ on day $4, P<0.05$ for all) as shown in Figure $1 \mathrm{~B}$.

Moreover, we compared the trends in total intake volume including infusion solution and water intake (Figure 1C and 1D). In HFpEF patients, total intake volume (days 1 and 2) and water intake (days 1, 2, 3, and 4) were higher in the tolvaptan group than in the carperitide group, however, infusion solution intake was higher in the carperitide group than in the tolvaptan group (days 3, 4, and 7) $(P<0.05$ for all) (Figure $1 C)$. On the other hand, in HFrEF patients, although total intake volume 
Table I. Comparisons of Clinical Characteristics Between the Preserved and Reduced Ejection Fraction Patients in the Tolvaptan and Carperitide Groups

\begin{tabular}{lcccc}
\hline & \multicolumn{2}{c}{ Tolvaptan $(n=54)$} & \multicolumn{2}{c}{ Carperitide $(n=54)$} \\
& $\operatorname{HFpEF}(n=25)$ & $\operatorname{HFrEF}(n=29)$ & $\operatorname{HFpEF}(n=17)$ & $\operatorname{HFrEF}(n=37)$ \\
\hline Age (years) & $79 \pm 9$ & $71 \pm 11$ & $80 \pm 8$ & $73 \pm 11$ \\
Gender (Male/Female) & $11 / 14$ & $18 / 11$ & $7 / 10$ & $25 / 12$ \\
NYHA functional class (II/III and IV) & $4 / 21$ & $7 / 22$ & $5 / 12$ & $6 / 31$ \\
Ischemic etiology, $n(\%)$ & $4(16)$ & $9(31)$ & $2(12)$ & $11(30)$ \\
Body weight (kg) & $58.9 \pm 12.0$ & $58.0 \pm 14.0$ & $54.0 \pm 10.1$ & $56.4 \pm 11.9$ \\
Heart rate (/minute) & $80 \pm 21^{\#}$ & $92 \pm 20$ & $70 \pm 15^{\#}$ & $86 \pm 23$ \\
Systolic blood pressure (mmHg) & $139 \pm 20$ & $124 \pm 26$ & $132 \pm 22$ & $127 \pm 29$ \\
Diastolic blood pressure (mmHg) & $77 \pm 15$ & $76 \pm 18$ & $73 \pm 19$ & $74 \pm 20$ \\
Echocardiography & & & & \\
LVEDD (mm) & $42 \pm 10^{\#}$ & $59 \pm 10$ & $46 \pm 7^{\#}$ & $56 \pm 7$ \\
LVEF (\%) & $63 \pm 7^{\#}$ & $31 \pm 10$ & $63 \pm 6^{\#}$ & $37 \pm 8$ \\
IVC (mm) & $14 \pm 6$ & $16 \pm 6$ & $14 \pm 7$ & $15 \pm 7$ \\
BNP ${ }^{*}$ (pg/mL) & $300.0(352.6)^{\#}$ & $993.0(1229.5)$ & $308.5(539.4)^{\#}$ & $860.0(1308.0)$ \\
Blood urea nitrogen (mg/dL) & $21.7 \pm 8.0$ & $26.8 \pm 19.1$ & $22.6 \pm 11.1$ & $25.8 \pm 13.5$ \\
Serum creatinine (mg/dL) & $1.00 \pm 0.41$ & $1.32 \pm 0.92$ & $0.97 \pm 0.38$ & $1.37 \pm 0.87$ \\
Estimated GFR (mL/minute/1.73 m $\left.{ }^{2}\right)$ & $53.9 \pm 18.3$ & $51.6 \pm 25.5$ & $57.9 \pm 26.7$ & $46.3 \pm 18.9$ \\
Serum sodium (mEq/L) & $141 \pm 4$ & $138 \pm 7$ & $141 \pm 3$ & $139 \pm 4$ \\
Serum potassium (mEq/L) & $4.2 \pm 0.4$ & $4.2 \pm 0.7$ & $4.0 \pm 0.5$ & $4.2 \pm 0.7$ \\
Concomitant medication, $n$ (\%) & & & & \\
$\quad$ Loop diuretics & $21(78)$ & $27(93)$ & $16(94)$ & $35(95)$ \\
Thiazide diuretics & $2(8)$ & $2(7)$ & $2(12)$ & $3(8)$ \\
Spironolactone & $16(64)$ & $14(48)$ & $12(71)$ & $21(57)$ \\
$\beta$-blocker & $9(36)^{\#}$ & $20(68)$ & $7(41)^{\#}$ & $27(73)$ \\
ACE inhibitors or ARBs & $13(52)$ & $10(34)$ & $10(59)$ & $21(57)$ \\
Ca-inhibitors & $6(24)$ & $3(10)$ & $1(6)$ & $5(14)$ \\
\hline
\end{tabular}

HFpEF indicates heart failure with preserved ejection fraction; HFrEF, heart failure with reduced ejection fraction; NYHA, New York Heart Association; LVEDD, left ventricular end diastolic diameter; LVEF, left ventricular ejection fraction; IVC, inferior vena cava; BNP, B-type natriuretic peptide; GFR, glomerular filtration rate; ACE, angiotensin converting enzyme; and ARB, angiotensin receptor blocker. "Skewed data are reported as median (inter-quartile range). ${ }^{~} P<0.05$ versus HFrEF in same group.

\section{Daily urine volume}

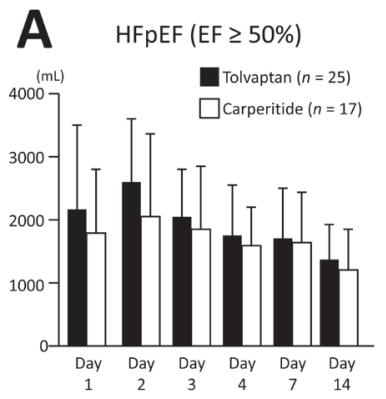

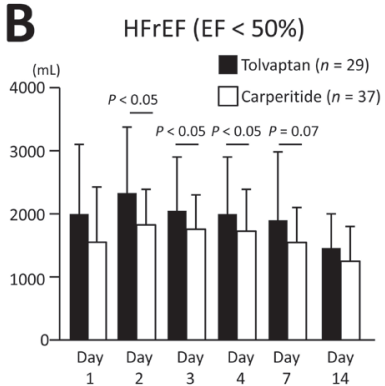

Total intake volume

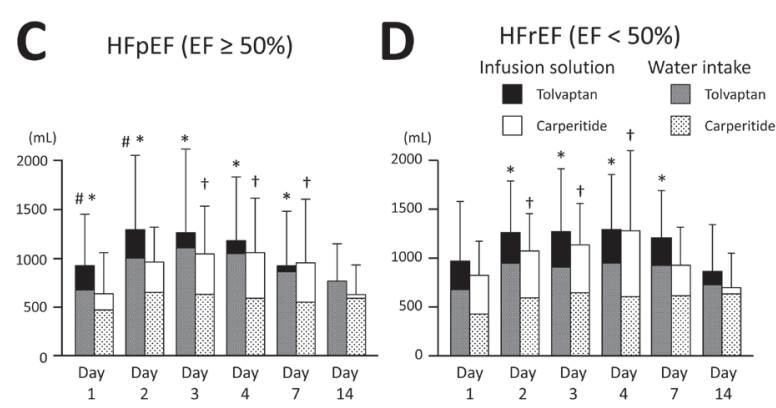

Figure 1. Comparisons of trends in urine volume between tolvaptan and carperitide group patients (A) with heart failure and preserved ejection fraction $(\mathrm{HFpEF})$ and $(\mathbf{B})$ with heart failure and reduced ejection fraction (HFrEF). Comparisons of trends in total intake volume including infusion solution and water intake between tolvaptan and carperitide group patients $(\mathbf{C})$ with $\mathrm{HFpEF}$ and $(\mathbf{D})$ with $\mathrm{HFrEF} .{ }^{\#} P<0.05$ versus carperitide group in total intake volume. ${ }^{*} P<0.05$ versus carperitide group in water intake. ${ }^{\dagger} P<0.05$ versus tolvaptan group in infusion solution.

was not different between the two groups, water intake was higher in the tolvaptan group than in the carperitide group on days $2,3,4$, and 7 , and infusion solution was higher in the carperitide group than in the tolvaptan group on days 2,3 , and $4(P<0.05$ for all) (Figure 1D).

Concomitant medications in the HFpEF and HFrEF patients in the tolvaptan and carperitide groups are presented in Table I. In both the tolvaptan and carperitide groups, $\beta$-blockers were more frequently given in HFrEF patients than in HFpEF patients $(P<0.05$ for all $)$.
Comparisons of daily urine volume based on blood pressure: In the tolvaptan group, there were 22 patients with high blood pressure $(\geq 140 \mathrm{mmHg}$ ) and 32 patients with low blood pressure $(<140 \mathrm{mmHg})$, whereas there were 17 patients with high blood pressure and 38 patients with low blood pressure in the carperitide group. Baseline clinical characteristics are shown in Table II. Left ventricular EF tended to be lower in low blood pressure than in high blood pressure patients in both the tolvaptan and carperitide groups. In high blood pressure patients, the daily urine volume showed no statistically signifi- 
Table II. Comparisons of Clinical Characteristics Between High and Low Blood Pressure Patients in the Tolvaptan and Carperitide Group

\begin{tabular}{lcccc}
\hline & \multicolumn{2}{c}{ Tolvaptan $(n=54)$} & \multicolumn{2}{c}{ Carperitide $(n=55)$} \\
& High BP $(n=22)$ & Low BP $(n=32)$ & High BP $(n=17)$ & Low BP $(n=38)$ \\
\hline Age (years) & $77 \pm 12$ & $72 \pm 11$ & $77 \pm 10$ & $75 \pm 11$ \\
Gender (Male/Female) & $11 / 11$ & $18 / 14$ & $12 / 5$ & $21 / 17$ \\
NYHA functional class (II/III and IV) & $4 / 18$ & $7 / 25$ & $3 / 14$ & $8 / 30$ \\
Body weight (kg) & $59.6 \pm 13.5$ & $58.2 \pm 12.9$ & $60.9 \pm 11.9$ & $53.9 \pm 10.9$ \\
Heart rate (/minute) & $82 \pm 22$ & $89 \pm 20$ & $87 \pm 25$ & $80 \pm 22$ \\
Systolic blood pressure (mmHg) & $156 \pm 17^{\#}$ & $113 \pm 15$ & $159 \pm 21^{\#}$ & $114 \pm 14$ \\
Diastolic blood pressure (mmHg) & $85 \pm 17^{\#}$ & $70 \pm 13$ & $86 \pm 20^{\#}$ & $68 \pm 16$ \\
Echocardiography & & & & \\
LVEDD (mm) & $48 \pm 11$ & $55 \pm 12$ & $54 \pm 6$ & $53 \pm 10$ \\
LVEF (\%) & $53 \pm 12$ & $42 \pm 19$ & $51 \pm 14$ & $41 \pm 15$ \\
$\quad$ IVC (mm) & $13 \pm 6$ & $17 \pm 6$ & $13 \pm 4$ & $16 \pm 7$ \\
BNP ${ }^{\text {(pg/mL) }}$ & $483.0(696.5)$ & $573.0(496.9)$ & $566.4(1178.2)$ & $599.0(712.5)$ \\
Blood urea nitrogen (mg/dL) & $25.0 \pm 17.5$ & $24.1 \pm 13.8$ & $27.0 \pm 14.9$ & $24.0 \pm 11.6$ \\
Serum creatinine (mg/dL) & $1.23 \pm 1.00$ & $1.14 \pm 0.54$ & $1.45 \pm 1.13$ & $1.15 \pm 0.50$ \\
Estimated GFR (mL/minute/1.73 m $\left.{ }^{2}\right)$ & $51.5 \pm 20.0$ & $53.3 \pm 24.0$ & $50.3 \pm 28.1$ & $49.9 \pm 18.8$ \\
Serum sodium (mEq/L) & $141 \pm 6$ & $138 \pm 6$ & $141 \pm 3$ & $140 \pm 4$ \\
Serum potassium (mEq/L) & $4.1 \pm 0.6$ & $4.3 \pm 0.6$ & $4.0 \pm 0.6$ & $4.2 \pm 0.6$ \\
Concomitant medication, $n(\%)$ & & & & \\
$\quad$ Loop diuretics & $19(86)$ & $29(83)$ & $16(94)$ & $35(92)$ \\
Thiazide diuretics & $1(5)$ & $3(9)$ & $4(24)^{\#}$ & $1(3)$ \\
Spironolactone & $11(50)$ & $19(59)$ & $8(47)$ & $25(66)$ \\
$\beta$-blocker & $10(45)$ & $19(59)$ & $11(65)$ & $24(63)$ \\
ACE inhibitors or ARBs & $11(50)$ & $12(38)$ & $10(59)$ & $21(55)$ \\
Ca-inhibitors & $5(23)$ & $4(13)$ & $4(24)$ & $2(6)$ \\
\hline BP & & & &
\end{tabular}

$\mathrm{BP}$ indicates blood pressure. "Skewed data are reported as median (inter-quartile range). ${ }^{\#} P<0.05$ versus low blood pressure in same group.
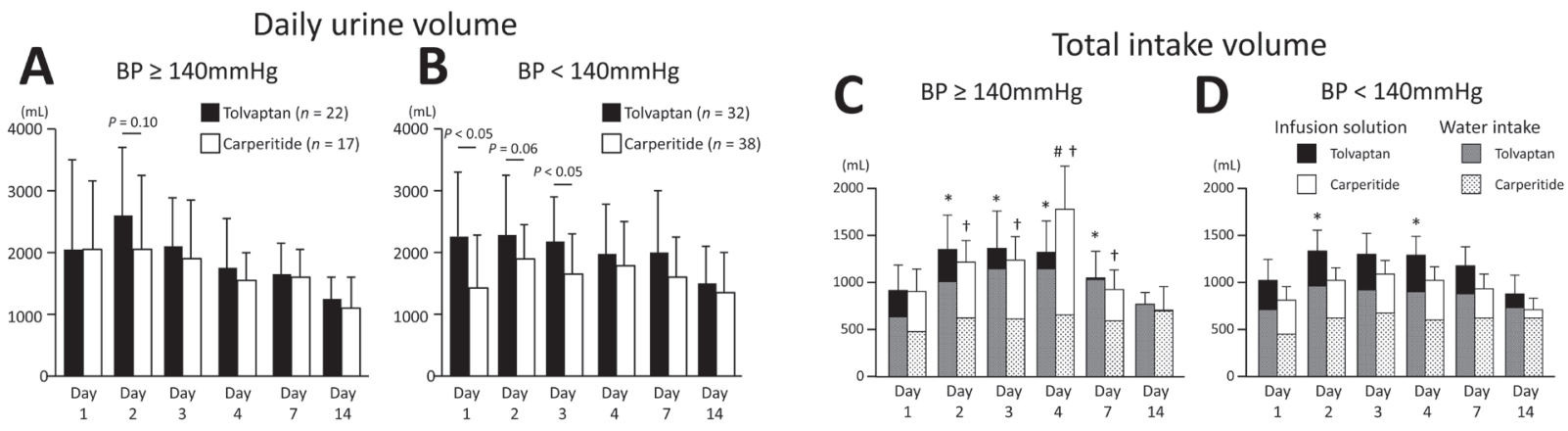

Figure 2. Comparisons of trends in urine volume between tolvaptan and carperitide groups (A) with blood pressure more than or equal to and (B) less than $140 \mathrm{mmHg}$. Comparisons of trends in total intake volume including infusion solution and water intake between tolvaptan and carperitide groups (C) with blood pressure more than or equal to and (D) less than $140 \mathrm{mmHg} .{ }^{\#} P<0.05$ versus carperitide group in total intake volume. ${ }^{*} P<0.05$ versus carperitide group in water intake. ${ }^{\dagger} P<0.05$ versus tolvaptan group in infusion solution.

cant difference between the tolvaptan and carperitide groups, however, it was significantly higher in the tolvaptan group on day $1(2094 \pm 1120 \mathrm{~mL}$ versus $1514 \pm 779 \mathrm{~mL}, P=0.021)$ and on day $3(2089 \pm 754 \mathrm{~mL}$ versus $1661 \pm 653 \mathrm{~mL}, P=0.017)$ than in the carperitide group among low blood pressure patients (Figure 2B).

As shown in Figures 2C and 2D, total intake volume was not different between the tolvaptan and carperitide groups in either the high or low blood pressure patients. However, water intake were higher in the tolvaptan group than in the carperitide group, and infusion solution intake was higher in the carperitide group than in the tolvaptan group on days 2, 3, 4, and $7(P<0.05$ for all) (Figure $2 \mathrm{C})$. On the other hand, in low blood pressure patients, although infusion solution intake was not different between the two groups, water intake was higher in the tolvaptan group than in the carperitide group on days 2 and 4 ( $P<0.05$ for all) (Figure 2D).

As shown in Table II, although thiazide diuretics were more frequently used in high blood pressure patients than in low blood pressure patients in the carperitide group, the use of loop diuretics, $\beta$-blockers, ACE inhibitors or ARBs, and Ca-inhibitors was not different between high and low blood pressure patients in the tolvaptan and carperitide groups.

Moreover, we analyzed patients with and without an intravenous vasodilator separately. Vasodilators were required by 6 and 7 ADHF patients in the tolvaptan and carperitide groups, 


\section{Daily urine volume}
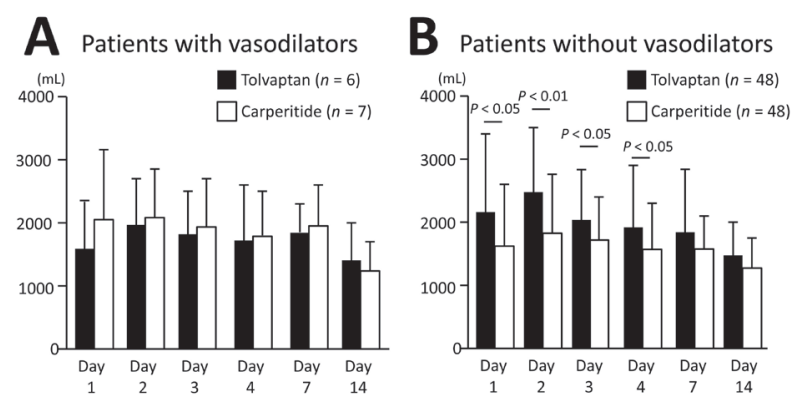

Figure 3. Comparisons of trends in urine volume between tolvaptan and carperitide groups (A) with and (B) without vasodilators.

respectively, and there were no significant differences in daily urine volume between the tolvaptan and carperitide groups (Figure 3A). In patients who did not require vasodilators for ADHF treatment, daily urine volumes were significantly higher in the tolvaptan group on day $1(2112 \pm 1284 \mathrm{~mL}$ versus $1591 \pm 941 \mathrm{~mL}, P=0.030)$, day $2(2418 \pm 1105 \mathrm{~mL}$ versus $1781 \pm 945 \mathrm{~mL}, P=0.004)$, day $3(2088 \pm 793 \mathrm{~mL}$ versus $1692 \pm 749 \mathrm{~mL}, P=0.002)$, and day $4(1952 \pm 885 \mathrm{~mL}$ versus $1586 \pm 731 \mathrm{~mL}, P=0.003$ ) than in the carperitide group (Figure $3 \mathrm{~B})$. There was no statistically significant difference in systolic blood pressure between the patients with and without an intravenous vasodilator (134 \pm 23 versus $126 \pm 31 \mathrm{mmHg}$ ).

Comparison of adverse events within carperitide group: In the present study, 8 adverse events requiring drug discontinuation occurred during the study period, and the details of these events were reported in our previous report. ${ }^{11)}$ There was only one event of hypernatremia in the tolvaptan group, however in the carperitide group there were 7 adverse events, the majority $(n=6)$ of which were cardiovascular events including hypotension and worsening heart failure due to hypotension. Although the number of cardiovascular events in $\operatorname{HFrEF}(n=5)$ and low blood pressure patients $(n=5)$ was greater than in HFpEF $(n=1)$ and high blood pressure patients $(n=1)$, the differences were not statistically significant.

\section{Discussion}

The present study revealed that tolvaptan was more effective than carperitide for the treatment of ADHF patients with reduced left ventricular systolic function and low blood pressure.

Clinical scenarios are useful criteria for treatment of $\mathrm{ADHF}$ because the respective scenarios reflect the pathology and characteristics of patients with ADHF. In clinical scenario 1 (systolic blood pressure $\geq 140 \mathrm{mmHg}$ ), dyspnea which is related to diffuse pulmonary edema typically develops abruptly, but relatively few systemic edemas are present, due to an acute elevation of filling pressure in the left ventricle and blood pressure. $^{14,17)}$ Therefore, one of the most useful treatments in this clinical scenario is vasodilation for reduction of blood pressure and left ventricular filling pressure, and diuretics are rarely indicated unless volume overload is present. Patients in clinical scenario 1 often have HFpEF. ${ }^{16)}$ In fact, although $56.4 \%$ of the patients in the high blood pressure group had preserved EF
$(22 / 39)$, only $31.4 \%$ of the patients in the low blood pressure group had reduced EF (22/70) in this study. In clinical scenario 2 (systolic blood pressure 100 to $140 \mathrm{mmHg}$ ), however, symptoms generally develop gradually compared to clinical scenario 1 , along with a progressive increase in body weight. ${ }^{16)}$ Congestion is predominated by systemic edema compared to pulmonary edema, and body fluid retention is more severe in clinical scenario 2 than in $1{ }^{16)}$ Therefore, the main therapeutic target in clinical scenarios 1 and 2 is considered to be different, and diuresis might be more important in clinical scenario 2. Systolic blood pressure was not significantly different with and without intravenous vasodilators. However, since the degree of blood pressure decrease was larger in the carperitide group than in the tolvaptan group, ${ }^{11)}$ the diuretic effect might be attenuated further by vasodilators in the carperitide group.

The incidence of hypotension was $9.09 \%$ in our AVCMA trial, ${ }^{11)}$ a percentage that was similar to another report in the "real world" of carperitide therapy $(9.45 \%) .{ }^{18)}$ Gheorghiade, et al reported that tolvaptan did not influence blood pressure in chronic heart failure patients, ${ }^{19)}$ and we previously reported that tolvaptan induced less reduction of blood pressure and cardiovascular adverse events despite inducing higher urine volume than carperitide. ${ }^{11)}$ The results of the present study suggest that cardiovascular adverse events in the carperitide group are prone to occur in patients with low blood pressure and low ejection fraction. Carperitide is not used in ADHF therapy in Western countries, and nesiritide, which is a recombinant form of human B-type natriuretic peptide, is more frequently used in HFrEF patients than in HFpEF patients. ${ }^{13)}$ However, our report might suggest that carperitide should be used in patients with high blood pressure or HFpEF, while tolvaptan should be used in patients without high blood pressure or HFrEF because tolvaptan was less likely to affect blood pressure. The effectiveness of tolvaptan in reduced systolic function was improved using Dahl salt-sensitive hypertensive rats. ${ }^{20)}$ Imamura, et al reported that the clinical symptom score, which was defined as an increase of urine volume after administration of tolvaptan, was improved in responders. ${ }^{21)}$ Moreover, carperitide is an intravenously administered natriuretic peptide but tolvaptan is an orally administered aquaretic drug and is therefore effective in patients who can take drugs orally and drink water, and especially in those with hyponatremia. Carperitide is effective in patients who are not eligible for oral administration due to intubation, or those who require an auxiliary circulation system such as an intra-aortic balloon pump or percutaneous cardiopulmonary support. We should use these drugs accordingly based on the pathology and characteristics of each ADHF patient.

In this study, tolvaptan could be considered as an effective and safe option, especially in patients with low cardiac function such as reduced ejection fraction and low blood pressure.

Study limitations: First, the administration routes and pharmacological mechanisms of these two drugs were different. There might have been a synergy effect if we had used tolvaptan and carperitide in combination. Second, the study drugs were randomly assigned, but not blinded, in this trial. Therefore, it is possible that any results of this study including adverse events might have been influenced by the lack of blinding. Third, the number of study subjects was small. In future research, a larger scale study will be necessary to validate 
the clinical usefulness of tolvaptan in Japan.

\section{APPENDiX}

Investigators and institutions participating in the acute heart failure volume control multicenter randomized (AVCMA) trial are shown below;

In addition to the authors, the following investigators participated in the AVCMA trial: Takamasa Sato, Takashi Owada, Yuichi Nakamura, Hiroyuki Yamauchi, Minoru Nodera, Atsuro Masuda, Tetsuro Yokokawa, Fukushima Medical University; Shigebumi Suzuki, Kazuyuki Yoshinari, Yasuyuki Watanabe, Hideaki Dairaku, Fukushima Accident Hospital; Kazuaki Tamagawa, Fukushima Prefectural Aizu General Hospital; Kenichi Watanabe, Takayuki Sakamoto, Fukushima Red Cross Hospital; Tomoyuki Watanabe, Health Co-op Watari Hospital; Hiroshi Ohtani, Iwase General Hospital; Nobuo Komatsu, Hiroto Takeda, Ohta Nishinouchi Hospital; Masahiko Sato, Katuya Ando, Public Soma General Hospital; Hideki Ohtake, Hirofumi Machii, Saiseikai Fukushima General Hospital; Masahiro Ono, Mitsuru Muto, Taku Ohsugi, Keiichi Kawamura, Wakako Naganuma, Southern Tohoku General Hospital; Tetsu Watanabe, Takuya Miyamoto, Takehiko Miyashita, Takanori Arimoto, Mitsunori Ishino, Hyuma Daidouji, Yoshinori Yashiro, Shintaro Sasaki, Daisuke Ishigaki, Yuki Honda, Yamagata University School of Medicine; Tomoyasu Yahagi, Yamagata Prefectural Central Hospital; Shigeo Sugawara, Kazuyoshi Kaneko, Toshiki Sasaki, Nihonkai General Hospital

\section{REFERENCES}

1. Hunt SA, Abraham WT, Chin MH, et al; American College of Cardiology Foundation; American Heart Association. 2009 Focused update incorporated into the ACC/AHA 2005 Guidelines for the Diagnosis and Management of Heart Failure in Adults A Report of the American College of Cardiology Foundation/American Heart Association Task Force on Practice Guidelines Developed in Collaboration With the International Society for Heart and Lung Transplantation. J Am Coll Cardiol 2009; 53: e1-e90.

2. Peacock WF, Costanzo MR, De Marco T, et al; ADHERE Scientific Advisory Committee and Investigators. Impact of intravenous loop diuretics on outcomes of patients hospitalized with acute decompensated heart failure: insights from the ADHERE registry. Cardiology 2009; 113: 12-9.

3. Adams KF Jr, Fonarow GC, Emerman CL, et al; ADHERE Scientific Advisory Committee and Investigators. Characteristics and outcomes of patients hospitalized for heart failure in the United States: rationale, design, and preliminary observations from the first 100,000 cases in the Acute Decompensated Heart Failure National Registry (ADHERE). Am Heart J 2005; 149: 209-16.

4. Abdel-Qadir HM, Tu JV, Yun L, Austin PC, Newton GE, Lee DS Diuretic dose and long-term outcomes in elderly patients with heart failure after hospitalization. Am Heart J 2010; 160: 264-71.

5. Eshaghian S, Horwich TB, Fonarow GC. Relation of loop diuretic dose to mortality in advanced heart failure. Am J Cardiol 2006; 97: 1759-64.

6. Sato N, Kajimoto K, Keida T, et al; TEND Investigators. Clinical features and outcome in hospitalized heart failure in Japan (from the ATTEND Registry). Circ J 2013; 77: 944-51.

7. Nomura F, Kurobe N, Mori Y, et al. Multicenter prospective investigation on efficacy and safety of carperitide as a first-line drug for acute heart failure syndrome with preserved blood pressure:
COMPASS: Carperitide Effects Observed Through Monitoring Dyspnea in Acute Decompensated Heart Failure Study. Circ J 2008; 72: 1777-86

8. Hata N, Seino Y, Tsutamoto T, et al. Effects of carperitide on the long-term prognosis of patients with acute decompensated chronic heart failure: the PROTECT multicenter randomized controlled study. Circ J 2008; 72: 1787-93.

9. Costello-Boerrigter LC, Smith WB, Boerrigter G, et al. Vasopressin-2-receptor antagonism augments water excretion without changes in renal hemodynamics or sodium and potassium excretion in human heart failure. Am J Physiol Renal Physiol 2006; 290: F273-8.

10. Gheorghiade M, Konstam MA, Burnett JC Jr, et al; Efficacy of Vasopressin Antagonism in Heart Failure Outcome Study With Tolvaptan (EVEREST) Investigators. Short-term clinical effects of tolvaptan, an oral vasopressin antagonist, in patients hospitalized for heart failure: the EVEREST Clinical Status Trials. JAMA 2007; 297: 1332-43.

11. Suzuki S, Yoshihisa A, Yamaki T, et al; AVCMA investigators. Acute heart failure volume control multicenter randomized (AVCMA) trial: comparison of tolvaptan and carperitide. J Clin Pharmacol 2013; 53: 1277-85.

12. Fonarow GC, Stough WG, Abraham WT, et al; OPTIMIZE-HF Investigators and Hospitals. Characteristics, treatments, and outcomes of patients with preserved systolic function hospitalized for heart failure: a report from the OPTIMIZE-HF Registry. J Am Coll Cardiol 2007; 50: 768-77.

13. Yancy CW, Lopatin M, Stevenson LW, et al; ADHERE Scientific Advisory Committee and Investigators. Clinical presentation, management, and in-hospital outcomes of patients admitted with acute decompensated heart failure with preserved systolic function: a report from the Acute Decompensated Heart Failure National Registry (ADHERE) Database. J Am Coll Cardiol 2006; 47: 76-84.

14. Gheorghiade M, Abraham WT, Albert NM, et al; OPTIMIZE-HF Investigators and Coordinators. Systolic blood pressure at admission, clinical characteristics, and outcomes in patients hospitalized with acute heart failure. JAMA 2006; 296: 2217-26.

15. Zannad F, Mebazaa A, Juillière Y, et al; EFICA Investigators. Clinical profile, contemporary management and one-year mortality in patients with severe acute heart failure syndromes: The EFICA study. Eur J Heart Fail 2006; 8: 697-705.

16. Mebazaa A, Gheorghiade M, Piña IL, et al. Practical recommendations for prehospital and early in-hospital management of patients presenting with acute heart failure syndromes. Crit Care Med 2008; 36: S129-39. (Review)

17. Kawaguchi M, Hay I, Fetics B, Kass DA. Combined ventricular systolic and arterial stiffening in patients with heart failure and preserved ejection fraction: implications for systolic and diastolic reserve limitations. Circulation 2003; 107: 714-20.

18. Suwa M, Seino Y, Nomachi Y, Matsuki S, Funahashi K. Multicenter prospective investigation on efficacy and safety of carperitide for acute heart failure in the 'real world' of therapy. Circ J 2005; 69: 283-90.

19. Gheorghiade M, Niazi I, Ouyang J, et al; Tolvaptan Investigators. Vasopressin V2-receptor blockade with tolvaptan in patients with chronic heart failure results from a double-blind, randomized trial. Circulation 2003; 107: 2690-6.

20. Ishikawa M, Kobayashi N, Sugiyama F, Onoda S, Ishimitsu T. Renoprotective effect of vasopressin v2 receptor antagonist tolvaptan in Dahl rats with end-stage heart failure. Int Heart J 2013; 54: 98-106.

21. Imamura T, Kinugawa K, Minatsuki S, et al. Tolvaptan can improve clinical course in responders. Int Heart J 2013; 54: 377-81. 